\title{
Parental feeding styles, young children's fruit, vegetable, water and sugar-sweetened beverage consumption, and the moderating role of maternal education and ethnic background
}

\author{
Maj-Britt MR Inhulsen ${ }^{1, *}$, Saskia YM Mérelle ${ }^{1, *}$ and Carry M Renders ${ }^{2}$ \\ 'GGD Kennemerland, Zijlweg 200, 2015 CK Haarlem, The Netherlands: ${ }^{2}$ Department of Health Sciences, Faculty \\ of Earth \& Life Sciences, Vrije Universiteit Amsterdam, Amsterdam Public Health Research Institute, Amsterdam, \\ The Netherlands
}

Submitted 21 July 2016: Final revision received 1 April 2017: Accepted 26 May 2017: First published online 17 July 2017

\begin{abstract}
Objective: To examine the associations between parental feeding styles and children's dietary intakes and the modifying effect of maternal education and children's ethnicity on these associations.

Design: Cross-sectional study of parental feeding styles, assessed by the Parental Feeding Style Questionnaire, and children's dietary intakes. Multiple regression analyses were carried out to assess the associations between the parental feeding styles studied ('control', 'emotional feeding', 'encouragement to eat' and 'instrumental feeding') and children's dietary intakes (consumption of fruit, vegetables, water and sugar-sweetened beverages (SSB)). The modifying effect of maternal education and children's ethnicity on these associations was explored. Setting: North-western part of the Netherlands.

Subjects: Children aged 3-7 years ( $n$ 5926).

Results: Both 'encouragement' and 'control' were associated with higher consumption of vegetables and lower consumption of SSB, but only 'encouragement' was positively associated with fruit and water intakes. 'Instrumental feeding' showed a positive association with SSB and negative associations with fruit, vegetable and water consumption. No significant associations were found for 'emotional feeding'. Maternal educational level and children's ethnicity moderated some associations; for example, 'control' was beneficial for vegetable intake in all subgroups, whereas the association with SSB was beneficial only in highly educated mothers.

Conclusions: The study shows that both encouraging and controlling feeding styles may improve children's dietary behaviour, while 'instrumental feeding' may have a detrimental effect. Furthermore, maternal educational level and children's ethnicity influence these associations. The study's findings could provide a basis for development of interventions to improve parental feeding styles.
\end{abstract}

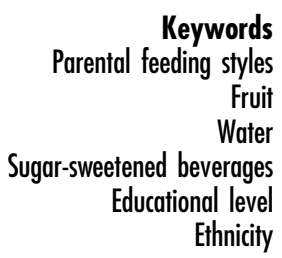

Important components of a healthy diet are the daily consumption of breakfast and a sufficient daily intake of fruit and vegetables, which are all associated with sustainable health benefits ${ }^{(1,2)}$. Dietary behaviour seems to persist from childhood into adulthood, which underlines the importance of a healthy diet during childhood ${ }^{(3,4)}$. Only $30 \%$ of Dutch children meet the recommendations for fruit consumption, while the percentage meeting the requirements for vegetable consumption is even lower $(6.5 \%)^{(5)}$. The daily recommendations for children aged 4-8 years are 1.5 pieces of fruit (100-150 g) and 2-3 serving spoons (100-150g) of vegetables ${ }^{(6)}$. Another important component of a healthy diet is the absence of sugarsweetened beverages (SSB) such as soft drinks and fruit drinks, since consumption of these beverages is positively associated with childhood overweight ${ }^{(7)}$.

The dietary behaviour of young children is determined by their environment, particularly by their family ${ }^{(8)}$. Children's ability to make healthful food choices is highly dependent on the food choices of their parents, since parents function as providers, enforcers and role models ${ }^{(8-11)}$. Moreover, parenting styles play a significant role in determining children's actual dietary intake and have also been shown to be associated with children's BMI ${ }^{(10,12)}$.

Children's dietary intake seems to be influenced not only by general parenting styles but also by parental feeding styles; that is, the way parents interact with their children when eating is concerned ${ }^{(10,13,14)}$. Analysis of 
clinical observations suggests that the following four parental feeding styles may be distinguished: (i) control over eating (parental control on food accessibility and moments of eating); (ii) emotional feeding (using food to modify a child's emotions); (iii) encouragement to eat (encouragement of the child's interest and curiosity, to get him/her to taste and eat a variety of foods); and (iv) instrumental feeding (using food to modify a child's behaviour) ${ }^{(15)}$. A study in 6- and 7-year-old Dutch children indicated that both 'instrumental feeding' and 'emotional feeding' may lead to an increase in children's snacking frequency ${ }^{(15)}$. In contrast, parental encouragement was associated with a reduction in children's consumption of sugar-sweetened and energy-dense food products ${ }^{(14)}$. Parental encouragement was also positively associated with fruit and vegetable intake ${ }^{(10,16-18)}$.

Little is known about the relationship between parental feeding styles and the dietary behaviour of children under the age of 6 years. The primary aim of the present study was therefore to assess the associations between parental feeding styles and the dietary intakes of young Dutch children. More specifically, associations of the abovementioned four parental feeding styles with the consumption of fruit, vegetables, water and SSB in children aged 3-7 years were determined. Previous studies indicated that maternal educational level and children's ethnic background were associated with both parenting styles and feeding styles $^{(9,19,20)}$. However, little is known about possible moderating effects of these sociodemographic characteristics on the associations between parental feeding styles and children's dietary intakes. The secondary aim of the study was therefore to explore what effect, if any, maternal educational level and children's ethnic background have on the associations between parental feeding styles and dietary intakes in the sample of young children studied here.

\section{Methods}

\section{Study population and procedures}

The present study makes use of data collected during the 'Kindermonitor' survey that was carried out in 2014 by the Municipal Health Services of Kennemerland, the Netherlands. The aim of that cross-sectional survey was to provide insight into the health status and health-related characteristics of young inhabitants of the Kennemerland region, which lies in north-western Netherlands. Participants were randomly sampled from the municipal registry. Parents or caregivers (referred to simply as 'parents' hereafter) of approximately 27000 children aged between 3 and 11 years were approached ${ }^{(21)}$. Data collection took place between October and December of 2014. In total, the parents of 10170 children participated in the survey (response rate $38 \%$ ). In the opinion of municipal youth health-care professionals, assessment of parental feeding styles was more relevant in children aged between
3 and 7 years, whereas more general parenting practices were more relevant in children aged between 8 and 11 years. In the range of interest to us (children aged 3-7 years), the parents of 6077 children participated in the survey (response rate 38\%). Of the 6077 questionnaires collected, sixty-seven were excluded from analysis because of incomplete information. An additional eighty-four questionnaires were excluded since children passed the age of 7 years during the data collection period, leaving a total of 5926 valid questionnaires for analysis. The data collection for the 'Kindermonitor' survey formed part of routine youth health care in the Netherlands. The approval of the medical ethics review board was therefore not required.

\section{Measurements}

\section{Children's dietary intake}

Fruit, vegetable, water and SSB consumption was assessed to determine children's dietary intakes. All variables were measured using a standardized questionnaire based on validated $\mathrm{FFQ}^{(22)}$.

Fruit and vegetables. Parents were asked to indicate how many days per week their child consumed fruit and vegetables. Response options for both ranged from '(almost) never' $(=1)$ to 'every day' $(=8)$. These variables were further specified by asking how many pieces of fruit and how many tablespoons of vegetables the child usually consumed per day. Response options for fruit consumption were: 'less than 1 piece', ' 1 piece', ' 2 pieces' and 'more than 2 pieces'. Response options for vegetable consumption were: 'less than 1 tablespoon', '1-2 tablespoons', '3-4 tablespoons', '5-6 tablespoons' and 'more than 6 tablespoons'. The means of both pieces of fruit and number of tablespoons of vegetables per day were calculated by multiplying the frequency of fruit and vegetable consumption by the amount of fruit and vegetable consumed and dividing by the total number of days per week. Therefore, fruit consumption of 'less than 1 piece' was recoded as ' 0.5 pieces' and 'more than 2 pieces' was

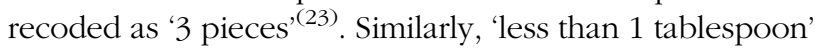
of vegetables was recoded as ' 0.5 tablespoons' and 'more than 6 tablespoons' as ' 7 tablespoons'. All other response options were coded as the mean of the reported number of tablespoons, that is $1.5,3.5$ and $5.5^{(23)}$. To determine whether children met the recommendations for vegetable intake, amounts specified in terms of tablespoons were converted into serving spoons assuming standard portions of $12 \mathrm{~g}$ (tablespoon) and $50 \mathrm{~g}$ (serving spoon).

Water and sugar-sweetened beverages. Parents were asked to indicate how many days per week their child consumed water or unsweetened tea and how many days per week their child consumed SSB including lemonade, soft drinks, fruit juice, sweet milk or yoghurt drinks and sugar-sweetened tea. Artificially sweetened drinks were not included. Response options for both water/ unsweetened tea and SSB consumption ranged from 
'(almost) never' $(=1)$ to 'every day' $(=8)$. These variables were further specified by asking how many glasses of water/unsweetened tea and SSB their child usually drank per day. Response options here were: 'none or less than 1 glass', '1 glass', '2 glasses', '3 glasses', '4 glasses' and '5 or more glasses'. Mean scores were obtained by multiplying the consumption frequency by the amount consumed and dividing by the total number of days per week. The item 'less than 1 glass' was recoded as ' 0.5 glasses' and ' 5 or more glasses' as '6 glasses',(23).

\section{Parental feeding styles}

Parental feeding styles were assessed with the aid of the validated Dutch version of the widely used Parental Feeding Style Questionnaire (PFSQ) ${ }^{(15)}$. Parents were asked to indicate how they dealt with feeding their child by stating to what extent they agreed with twenty-seven statements on a 5-point Likert scale with response options ranging from 'never' $(=1)$ to 'always' $(=5)$. The statements were related to the four above-mentioned parental feeding styles: control over eating (e.g. 'I decide how many snacks my child should have'), emotional feeding (e.g. 'I give my child something to eat to make him/her feel better when he/she is upset'), encouragement to eat (e.g. 'I praise my child if he/she eats what I give him/her') and instrumental feeding (e.g. 'I reward my child with something to eat when he/she is well-behaved') ${ }^{(15)}$. A mean score was calculated for each subscale, where a higher score indicates more frequent use of the feeding style in question. Cronbach's $\alpha$ was used to assess the internal consistency of the four PFSQ subscales in the present study population. The values found, 0.74, 0.83, 0.73 and 0.70 respectively, indicate that the internal consistency was acceptable or good ${ }^{(24)}$.

\section{Demographics}

Both parents were asked to report their highest completed level of education. The classification defined by Statistics Netherlands was used to categorize three educational levels: 'low' (primary school and lower vocational or lower general secondary education), 'medium' (intermediate vocational education, higher general secondary education and academic secondary education leading to university) and 'high' (higher vocational education and university) ${ }^{(25)}$. Since $85 \%$ of the questionnaires were filled in by the child's mother or female caregiver and $4 \%$ of the questionnaires failed to give the educational level of the father or male caregiver, the educational level of the mother was taken into account during analysis. To assess children's ethnicity, both parents were asked to report their country of origin, as well as the country of origin of their child. Following the definitions given by Statistics Netherlands, ethnicity was divided into 'non-native/non-Western', 'non-native/Western' or 'native Dutch"(26). Children were classified as non-native/ non-Western if at least one parent was born in Africa, Latin America, Asia (excluding Indonesia and Japan) or Turkey.
Children with at least one parent born outside the Netherlands, but inside Europe (excluding Turkey) or North America, Oceania or Indonesia or Japan, were classified as non-native/Western. Children were classified as native Dutch if both parents were born in the Netherlands ${ }^{(26)}$.

\section{Statistical analysis}

All analyses were performed using the statistical software package IBM SPSS Statistics version 23.0 and a significance level of $P<0.05$ was used in all analyses. Dietary intakes of fruit, vegetables, water and SSB were used as dependent variables. Parental feeding styles ('control', 'emotional feeding', 'encouragement' and 'instrumental feeding') were used as independent variables. The assumptions of normality and homoscedasticity in linear regression analysis were checked and a variance inflation factor of 0.7 was taken as the threshold in checks for multicollinearity ${ }^{(27)}$. Descriptive analysis was followed by multiple regression analysis to assess the relationship between parental feeding styles and children's dietary intakes. All parental feeding styles were entered simultaneously into the model for each dietary outcome. Analyses took demographic characteristics into account, to assess the impact of parental feeding styles on dietary intakes independent of the influence of demographics. ANOVA was then used to test group differences between maternal educational level and children's ethnicity for the variables parental feeding styles and dietary intakes. The possible moderating effect of maternal educational level and children's ethnicity on the associations between parental feeding styles and children's dietary intakes was assessed as the next step ${ }^{(9,19,20)}$. To this end, the associations for subgroups of maternal educational level (low; medium; high) and children's ethnicity (non-native/non-Western; non-native/Western; native Dutch) were estimated by simultaneously adding all interaction terms for educational level or ethnicity to the adjusted model of parental feeding styles with the outcome of interest. Analyses were adjusted for sociodemographic factors.

\section{Results}

Demographic characteristics and mean parental feeding style and dietary intake scores for the whole study sample ( $n$ 5926) are presented in Table 1 . Both genders were more or less evenly represented ( $51.3 \%$ boys and $48.7 \%$ girls) and the mean age of the participating children was 4.7 (1.5) years. The majority of children were of native Dutch origin (78.3\%) and most of the participating mothers were highly educated (56.4\%). Regarding parental feeding styles, parents were found to use 'control' and 'encouragement' the most frequently and 'instrumental feeding' and 'emotional feeding' the least. Children consumed on average 1.3 pieces of fruit and 2.9 tablespoons of vegetables daily. Daily recommendations on fruit and vegetable intake were met by 
35.5 and $22.4 \%$ of the children, respectively. Children's mean intake of SSB was $2 \cdot 0$ glasses/d, slightly higher than the consumption of water or unsweetened tea which was 1.7 glasses/d.

Table 2 presents the results of the adjusted multiple regression analysis of the association between each parental feeding style and each child dietary intake. Table 3 presents the distribution of parental feeding styles and children's dietary intakes across maternal educational level and children's ethnicity, whereas Tables 4 and 5 present the results for the associations between parental feeding styles and children's dietary intakes stratified by

Table 1 Characteristics of the study population of 3- to 7-year-old children and their parents ( $n$ 5926), north-western Netherlands, October-December 2014

\begin{tabular}{|c|c|c|c|c|}
\hline Variable & $n$ & $\%$ & Mean & SD \\
\hline Gender & 5923 & & & \\
\hline Boys & 3036 & $51 \cdot 3$ & - & - \\
\hline Girls & 2887 & $48 \cdot 7$ & - & - \\
\hline Age & 5926 & & 4.7 & 1.5 \\
\hline 3 years & 1804 & $30 \cdot 4$ & - & - \\
\hline 4 years & 1092 & $18 \cdot 4$ & - & - \\
\hline 5 years & 1013 & $17 \cdot 1$ & - & - \\
\hline 6 years & 972 & $16 \cdot 4$ & - & - \\
\hline 7 years & 1045 & $17 \cdot 6$ & - & - \\
\hline Children's ethnicity & 5926 & & & \\
\hline Non-native/non-Western & 718 & $12 \cdot 1$ & - & - \\
\hline Non-native Western & 570 & $9 \cdot 6$ & - & - \\
\hline Native Dutch & 4638 & $78 \cdot 3$ & - & - \\
\hline Educational level (mother) & 5846 & & & \\
\hline Low & 734 & $12 \cdot 5$ & - & - \\
\hline Medium & 1824 & $31 \cdot 1$ & - & - \\
\hline High & 3306 & $56 \cdot 4$ & - & - \\
\hline \multicolumn{5}{|l|}{ Parental feeding styles* } \\
\hline Control over eating & 5846 & - & $4 \cdot 3$ & 0.4 \\
\hline Emotional feeding & 5844 & - & 1.4 & 0.5 \\
\hline Encouragement to eat & 5846 & - & 4.0 & 0.5 \\
\hline Instrumental feeding & 5842 & - & 1.8 & 0.7 \\
\hline \multicolumn{5}{|c|}{$\begin{array}{l}\text { Dietary intake (based on weekly frequency } \\
\text { and usual amount consumed) }\end{array}$} \\
\hline Fruit (pieces/d) & 5901 & - & 1.3 & 0.7 \\
\hline Vegetables (tablespoons/d) & 5909 & - & $2 \cdot 9$ & $1 \cdot 8$ \\
\hline Water (glasses/d) & 5891 & - & 1.7 & $1 \cdot 3$ \\
\hline SBB (glasses/d) & 5903 & - & $2 \cdot 0$ & 1.3 \\
\hline \multicolumn{5}{|l|}{ Compliance with daily recommendations } \\
\hline Fruit intake ( $\geq 1.5$ pieces $/ \mathrm{d})$ & 5901 & 35.5 & - & - \\
\hline Vegetable intake ( $\geq 2-3$ serving spoons/d) & 5903 & 22.4 & - & - \\
\hline
\end{tabular}

SSB, sugar-sweetened beverages.

${ }^{*}$ Score on a 5-point Likert scale. subgroup of maternal educational level and children's ethnicity.

\section{Associations between parental feeding styles and children's dietary intakes}

The results of Table 2 show that both 'encouragement' and 'control' improved children's consumption of vegetables and reduced their consumption of SSB, but only 'encouragement' was associated with improved fruit and water intakes. In addition, 'instrumental feeding' promoted SSB consumption and led to lower consumption of fruit, vegetables and water. Furthermore, 'emotional feeding' was not significantly related to children's diet.

\section{Moderation by maternal educational level}

Table 3 presents the distribution of parental feeding styles and children's dietary intakes across maternal educational level. The differences between all parental feeding styles and dietary intake subgroups were significant. When stratified according to maternal educational level as presented in Table 4, 'control' was significantly associated with lower fruit consumption only for children whose mothers had a low educational level. Vegetable consumption was positively associated with 'control' in all educational level subgroups. Furthermore, 'control' was significantly associated with lower water consumption only in children whose mothers had a medium educational level and with lower SSB consumption only in children whose mothers had a high educational level. 'Emotional feeding' was significantly associated with higher vegetable consumption in children only when the mother had a low educational level. 'Encouragement' was significantly associated with higher fruit consumption and higher water consumption in all educational level subgroups. However, only in children whose mothers had a medium educational level was 'encouragement' also significantly associated with higher vegetable consumption and lower SSB consumption. 'Instrumental feeding' was significantly associated with lower fruit consumption in children whose mothers had a medium or high educational level, and with lower vegetable consumption in children whose mothers had a low educational level. Furthermore, it was significantly negatively associated with water consumption for children whose mothers had a medium or high educational level.

Table 2 Associations between parental feeding styles and 3- to 7-year-old children's daily consumption of fruit, vegetables, water and sugar-sweetened beverages (SSB), north-western Netherlands, October-December 2014

\begin{tabular}{lcccc}
\hline & Fruit (pieces; $\beta$ ) & Vegetables (tablespoons; $\beta$ ) & Water (glasses; $\beta$ ) & SSB (glasses; $\beta$ ) \\
\hline Parental feeding style & & & & $-0.04^{\star \star}$ \\
'Control' & -0.02 & $0.09^{\star \star \star}$ & -0.03 & 0.00 \\
'Emotional feeding' & 0.02 & 0.02 & -0.02 & $-0.05^{\star \star}$ \\
'Encouragement' & $0.11^{\star \star \star}$ & $0.05^{\star \star}$ & $0.14^{\star \star \star}$ & $0.03^{\star}$ \\
'Instrumental feeding' & $-0.07^{\star \star *}$ & $-0.05^{\star \star}$ & $-0.07^{\star \star}$ & 0 \\
\hline
\end{tabular}

$\beta$, standardized regression coefficient

Model adjusted for children's gender, age and ethnicity, and maternal educational level.

${ }^{\star} P<0.05,{ }^{* \star} P<0.01,{ }^{* \star *} P<0.001$. 
Table 3 Distribution of parental feeding styles and 3- to 7-year-old children's dietary intakes across maternal educational level and children's ethnicity, north-western Netherlands, October-December 2014

\begin{tabular}{|c|c|c|c|c|c|c|c|c|}
\hline & \multicolumn{2}{|c|}{ 'Control' } & \multicolumn{2}{|c|}{ 'Emotional feeding' } & \multicolumn{2}{|c|}{ 'Encouragement' } & \multicolumn{2}{|c|}{ 'Instrumental feeding } \\
\hline & Mean & SD & Mean & SD & Mean & SD & Mean & $\mathrm{SD}$ \\
\hline \multicolumn{9}{|l|}{ Maternal educational level } \\
\hline Low & 4.2 & 0.5 & 1.6 & 0.7 & 3.9 & 0.6 & 1.9 & 0.8 \\
\hline Medium & $4 \cdot 3$ & 0.4 & 1.4 & 0.5 & $4 \cdot 0$ & 0.5 & 1.8 & 0.7 \\
\hline High & 4.3 & 0.4 & 1.4 & 0.5 & $4 \cdot 1$ & 0.5 & 1.8 & 0.6 \\
\hline \multicolumn{9}{|l|}{ Children's ethnicity } \\
\hline Non-native/non-Western & $4 \cdot 1$ & 0.6 & 1.8 & 0.8 & 3.9 & 0.6 & $2 \cdot 1$ & 0.8 \\
\hline Non-native Western & 4.2 & 0.5 & 1.5 & 0.6 & 4.0 & 0.6 & 1.9 & 0.7 \\
\hline \multirow[t]{3}{*}{ Native Dutch } & 4.4 & 0.4 & 1.4 & 0.4 & $4 \cdot 0$ & 0.5 & $1 \cdot 8$ & 0.6 \\
\hline & \multicolumn{2}{|c|}{ Fruit (pieces/d) } & \multicolumn{2}{|c|}{ Vegetables (tablespoons/d) } & \multicolumn{2}{|c|}{ Water (glasses/d) } & \multicolumn{2}{|c|}{ SSB (glasses/d) } \\
\hline & Mean & SD & Mean & SD & Mean & SD & Mean & SD \\
\hline \multicolumn{9}{|l|}{ Maternal educational level } \\
\hline Low & $1 \cdot 3$ & 0.8 & 2.9 & $1 \cdot 8$ & $1 \cdot 7$ & 1.5 & $2 \cdot 2$ & 1.5 \\
\hline Medium & 1.2 & 0.7 & 2.8 & 1.7 & 1.6 & 1.3 & $2 \cdot 3$ & 1.4 \\
\hline High & 1.4 & 0.7 & 3.0 & 1.8 & 1.8 & 1.3 & 1.9 & 1.2 \\
\hline \multicolumn{9}{|l|}{ Children's ethnicity } \\
\hline Non-native/non-Western & 1.5 & 0.8 & 3.3 & $2 \cdot 0$ & $2 \cdot 0$ & 1.4 & 1.7 & 1.3 \\
\hline Non-native Western & 1.5 & 0.7 & 3.4 & $2 \cdot 0$ & $2 \cdot 1$ & 1.5 & 1.6 & $1 \cdot 3$ \\
\hline Native Dutch & 1.3 & 0.6 & 2.8 & 1.7 & 1.6 & 1.3 & $2 \cdot 1$ & 1.3 \\
\hline
\end{tabular}

SSB, sugar-sweetened beverages.

Table 4 Associations of parental feeding styles with 3- to 7-year-old children's dietary intakes, stratified by maternal educational level, north-western Netherlands, October-December 2014

\begin{tabular}{|c|c|c|c|c|}
\hline Feeding style/Maternal educational level & $\begin{array}{c}\text { Fruit } \\
\text { (pieces; } \beta \text { ) }\end{array}$ & $\begin{array}{c}\text { Vegetables } \\
\text { (tablespoons; } \beta \text { ) }\end{array}$ & $\begin{array}{c}\text { Water } \\
\text { (glasses; } \beta \text { ) }\end{array}$ & $\begin{array}{c}\text { SSB } \\
\text { (glasses; } \beta \text { ) }\end{array}$ \\
\hline \multicolumn{5}{|l|}{ ‘Control' } \\
\hline Low & $-0.13^{* *}$ & $0.14^{\star * *}$ & -0.03 & -0.04 \\
\hline Medium & -0.01 & $0.08^{\star *}$ & $-0.08^{\star \star}$ & -0.00 \\
\hline High & 0.01 & 0.08 *** & 0.01 & $-0.06^{\star \star}$ \\
\hline \multicolumn{5}{|l|}{ ‘Emotional feeding' } \\
\hline Low & -0.05 & $0.17^{\star \star \star}$ & 0.01 & -0.07 \\
\hline Medium & 0.04 & -0.01 & -0.05 & 0.00 \\
\hline High & 0.02 & -0.02 & -0.02 & 0.04 \\
\hline \multicolumn{5}{|l|}{ ‘Encouragement’ } \\
\hline Low & $0.12^{\star \star \star}$ & 0.06 & $0.10^{\star *}$ & -0.04 \\
\hline Medium & $0.11^{\star \star *}$ & $0.05^{\star}$ & $0 \cdot 17^{\star \star \star}$ & $-0.08^{\star *}$ \\
\hline High & $0.11^{\star * \star}$ & 0.04 & $0.12^{\star \star *}$ & -0.02 \\
\hline \multicolumn{5}{|l|}{ 'Instrumental feeding' } \\
\hline Low & 0.02 & $-0 \cdot 10^{*}$ & -0.05 & 0.03 \\
\hline Medium & $-0 \cdot 10^{\star \star \star}$ & -0.04 & $-0.09^{\star \star}$ & 0.05 \\
\hline High & $-0.07^{\star *}$ & -0.04 & $-0.05^{\star}$ & 0.02 \\
\hline
\end{tabular}

$\beta$, standardized regression coefficient; SSB, sugar-sweetened beverages.

Stratified analyses for maternal educational level, adjusted for children's gender, age and ethnicity.

${ }^{\star} P<0.05,{ }^{\star \star} P<0.01,{ }^{\star \star \star} P<0.001$.

\section{Moderation by children's ethnicity}

Table 3 presents the distribution of parental feeding styles and children's dietary intakes across children's ethnicity. Once again, the differences between all subgroups were significant. When stratified by children's ethnicity as shown in Table 5, 'control' was significantly associated with higher vegetable consumption in all ethnicity subgroups, but was significantly associated with lower SSB intake only in native Dutch children. 'Emotional feeding' was found to be significantly associated with higher fruit consumption in children with non-native/Western ethnicity and with higher vegetable consumption in children with non-native/non-Western ethnicity. It was also significantly associated with lower water consumption in native Dutch children. 'Encouragement' was found to be significantly related to an increase in fruit consumption only in children with non-native/non-Western or native Dutch ethnicity, to higher vegetable consumption among non-native/Western and native Dutch children, to higher water consumption in children with non-native/nonWestern or native Dutch ethnicity, and to lower SSB consumption in native Dutch children. 'Instrumental feeding' was significantly negatively associated with fruit consumption in children with non-native/Western 
Table 5 Associations of parental feeding styles with 3- to 7-year-old children's dietary intakes, stratified by children's ethnicity, north-western Netherlands, October-December 2014

\begin{tabular}{|c|c|c|c|c|}
\hline Feeding style/Children's ethnicity & $\begin{array}{c}\text { Fruit } \\
\text { (pieces; } \beta \text { ) }\end{array}$ & $\begin{array}{c}\text { Vegetables } \\
\text { (tablespoons; } \beta \text { ) }\end{array}$ & $\begin{array}{c}\text { Water } \\
\text { (glasses; } \beta \text { ) }\end{array}$ & $\begin{array}{c}\text { SSB } \\
\text { (glasses; } \beta \text { ) }\end{array}$ \\
\hline \multicolumn{5}{|l|}{ ‘Control' } \\
\hline Non-native/non-Western & -0.01 & $0 \cdot 11^{\star *}$ & -0.04 & -0.05 \\
\hline Non-native/Western & 0.02 & $0.09^{*}$ & 0.01 & -0.04 \\
\hline Native Dutch & -0.02 & $0.08^{\star \star *}$ & -0.03 & $-0.04^{*}$ \\
\hline \multicolumn{5}{|l|}{ ‘Emotional feeding' } \\
\hline Non-native/non-Western & 0.04 & $0.13^{\star \star \star}$ & 0.04 & -0.07 \\
\hline Non-native/Western & $0 \cdot 19^{\star \star \star}$ & 0.05 & 0.02 & 0.01 \\
\hline Native Dutch & -0.02 & -0.03 & $-0.06^{\star \star}$ & 0.03 \\
\hline \multicolumn{5}{|l|}{ ‘Encouragement’ } \\
\hline Non-native/non-Western & $0 \cdot 11^{\star \star}$ & 0.00 & $0.13^{\star \star \star}$ & -0.01 \\
\hline Non-native/Western & 0.07 & $0.09^{*}$ & 0.08 & 0.06 \\
\hline Native Dutch & $0.11^{\star \star \star}$ & $0.05^{\star \star}$ & $0.15^{\star \star *}$ & $-0.07^{\star \star *}$ \\
\hline \multicolumn{5}{|l|}{ 'Instrumental feeding' } \\
\hline Non-native/non-Western & -0.03 & $-0.09^{*}$ & $-0.09^{*}$ & 0.06 \\
\hline Non-native/Western & $-0.16^{\star \star}$ & $-0 \cdot 11^{*}$ & -0.09 & -0.01 \\
\hline Native Dutch & $-0.06^{\star \star}$ & -0.03 & $-0.06^{\star *}$ & $0.04^{*}$ \\
\hline
\end{tabular}

$\beta$, standardized regression coefficient; SSB, sugar-sweetened beverages.

Stratified analyses for children's ethnicity, adjusted for children's gender and age, and maternal educational level.

${ }^{\star} P<0.05,{ }^{\star \star} P<0.01,{ }^{\star \star \star} P<0.001$.

ethnicity and in native Dutch children, with vegetable consumption in non-native/non-Western and non-native/ Western children, and with water consumption in children with non-native/non-Western or native Dutch ethnicity. It was significantly associated with higher SSB consumption in native Dutch children only.

\section{Discussion}

The primary aim of the present cross-sectional study was to provide insight into the associations between parental feeding styles and dietary intakes in children aged 3-7 years. The secondary aim was to explore the modifying effects of maternal educational level and children's ethnic background on these associations.

Four parental feeding styles were investigated. 'Control' was found to be associated with a higher consumption of vegetables and a lower consumption of SSB in the overall study population. 'Encouragement' was also associated with higher consumption of vegetables and lower consumption of SSB, as well as with higher fruit and water intakes. In contrast, 'instrumental feeding' had an adverse influence on all types of dietary behaviour studied. 'Emotional feeding' was the only one that showed no significant association with any type of dietary behaviour studied in the total study population.

The associations between parental feeding styles and children's dietary behaviour were influenced by maternal educational level and by children's ethnic background. 'Encouragement' improved children's dietary behaviour in most subgroups and had a particularly favourable effect on fruit and water consumption in children with mothers of all educational levels. The reduction in SSB consumption due to this feeding style was particularly marked in children whose mother had a medium educational level and in native Dutch children. 'Control' showed beneficial associations with vegetable intake for all subgroups, but had more impact in children whose mother had a low educational level and in non-native/non-Western children. A relationship between 'control' and lower SSB consumption was found only in children whose mother had a high educational level and in native Dutch children. 'Instrumental feeding' gave rise to inconsistent findings, but had a mainly unfavourable effect on fruit and water consumption in children whose mother had a medium or high educational level and in native Dutch children. Unfavourable effects on fruit and vegetable consumption were found in non-native/ Western children. Although 'emotional feeding' was not associated with dietary intake in the overall study population, it was associated with higher vegetable consumption in children whose mother had a low educational level and in non-native/non-Western children, and with higher fruit consumption in non-native/Western children.

\section{Discussion of main findings}

In general, the mean scores found in the present study for all parental feeding styles were practically identical to those reported in previous studies on older children in the $6-8$ years age range ${ }^{(14,16)}$. These similarities indicate that parental feeding styles may be stable over time, at least for children between 3 and 8 years of age. The proportion of children in the present study who met Dutch recommendations for fruit consumption was $35.5 \%$, while a lower proportion, $22.4 \%$, met the requirements for vegetable consumption. Although both these figures are higher than the values found in a Dutch national survey on children aged 2-6 years, it will be noted that more than half the children participating in the present study did not meet the national recommendations ${ }^{(28)}$. 
The findings of the present study indicate that 'encouragement' tends to promote a healthy diet in children, showing significant favourable associations with all four outcomes and particularly with fruit and water consumption. It seems that children who are encouraged to eat a variety of foods consume more fruit and vegetables; this is in agreement with the findings of other studies ${ }^{(17,18,29)}$. Furthermore, previous research showed that encouraging children to adopt a healthy diet by offering them a variety of healthy foods stimulates their appetite and thus tends to increase their overall consumption ${ }^{(30,31)}$. This increased consumption is also seen in children who are repeatedly exposed to healthy foods, which contributes to their acceptance of such foods ${ }^{(30)}$. In addition, the present study found a negative association between 'encouragement' and children's SSB consumption. It is not yet clear whether the parents in question actively encouraged their child to consume less SSB or whether they encouraged their children to drink water instead. Another possibility is that the parents combined both these approaches, encouraging their children to replace SSB by water; this could then be seen as authoritative parenting ${ }^{(32)}$.

The present study showed that a controlling feeding style contributed to a healthy dietary intake regarding both vegetable and SSB consumption. One of the few studies that also examined the relationships between parental feeding styles and dietary intakes in a sample of young children found a comparable association between 'control' and meeting daily vegetable intake recommendations ${ }^{(18)}$. It seems that parental control of the accessibility of vegetables and the time when they are eaten, which are inherent to the concept of 'control' as measured by the PFSQ, is important for determining children's vegetable consumption. This is in line with the recommendation that parents should provide a variety of vegetables - without, however, forcing their child to eat them, as this might lead to a dislike for these foods ${ }^{(33)}$. Furthermore, as far as we know, the present study is the first to find that 'control' was significantly associated with a lower intake of SSB in young children. A previous study found a comparable negative association between 'control' and consumption of SSB and sweets rather than SSB alone ${ }^{(18)}$. Other studies found a similar reduction in soft drink consumption, but the effects they reported were not significant, probably due to small sample sizes ${ }^{(14,29)}$. The present study found that 'control' was not significantly associated with higher fruit consumption, in contrast to the results of comparable previous research ${ }^{(18,29)}$.

The present study indicates that 'instrumental feeding' is not conducive to a healthy diet in children, since it was associated with decreases in the amounts of fruit, vegetables and water that children consumed and with an increase in children's consumption of SSB. This confirms previous findings that 'instrumental feeding', or using food as a reward, is positively related to the consumption of unhealthy foods and beverages ${ }^{(14,18,20,23,34)}$. When parents respond to their children's behaviour by using rewards, sweet, salty and energy-rich foods are an obvious choice since there is evidence that these are in line with children's genetically predetermined predispositions ${ }^{(35)}$. This explains why 'instrumental feeding' tends to have an adverse effect on children's fruit, vegetable and water consumption, since these foods are known to be not sweet, salty or energy-rich which makes these foods probably less attractive as instruments for regulating a child's behaviour ${ }^{(35,36)}$.

On the other hand, the present study found no significant association between 'emotional feeding' in general and children's dietary intakes. This conflicts somewhat with the results of previous studies, which reported a positive relationship between this parental feeding style and children's snacking behaviour, consumption of energy-rich foods and, to a lesser extent, a reduction in fruit consumption in the long term ${ }^{(14,18,20,23,29)}$.

Finally, the present study found that the associations between parental feeding styles and children's dietary intakes tend to be influenced by both maternal educational level and children's ethnicity.

As far as maternal educational level is concerned, 'encouragement' was found to improve children's dietary behaviour and particularly to stimulate fruit and water consumption in children with mothers of all educational levels. The association with lower SSB consumption was only found in children whose mother had a medium educational level. This disagrees with the results of a previous study, which did not report any differences in the use of 'encouragement' between mothers with different levels of education ${ }^{(19)}$. 'Control' seems to be beneficial for children's vegetable intake irrespective of the mother's educational level, but to have more impact when the mother has a low educational level. A favourable association between 'control' and children's SSB consumption was found only when the mother had a high educational level, possibly because these parents are more aware of the adverse effects of SSB consumption. A previous study reported differences in the use of 'control' between mothers with different educational levels, but did not assess the effects of parental feeding styles on children's dietary behaviour ${ }^{(19)}$. The negative associations between 'instrumental feeding' and children's dietary intakes were moderated by maternal educational level. This feeding style is mainly unfavourably associated with fruit and water consumption in children whose mother has a medium or high educational level. Previous studies reported inconsistent results of the use of instrumental feeding' on children whose mothers had different levels of education $^{(19,20)}$. As no significant associations were found for 'emotional feeding' in general, hardly any of these associations appeared to be moderated by maternal educational level. The only exception was the positive association with children's vegetable consumption, which was found 
only when the mother had a low educational level. Previous studies also reported differences in maternal educational level and the use of 'emotional feeding', this feeding style being used less often by highly educated mothers ${ }^{(20)}$. This last finding is confirmed by the results of our study.

Regarding children's ethnicity, 'encouragement' was beneficial for children's dietary behaviour in most subgroups. The association with lower SSB consumption was found only in native Dutch children. 'Control' seemed to be beneficial for children's vegetable intake in all ethnicity subgroups, but had more impact in children with non-native/non-Western ethnicity. The negative associations between 'instrumental feeding' and children's dietary intake were moderated by children's ethnicity, fruit and vegetable consumption being particularly low in non-native/Western children. Most associations with 'emotional feeding' appeared not to be moderated by children's ethnicity. However, this feeding style was associated with higher fruit consumption in non-native/ Western children and with higher vegetable consumption in non-native/non-Western children.

Little has been published to date on feeding behaviour in response to children's emotions. Additional research is needed in this field, particularly on the modifying effect of maternal educational level and children's ethnicity in these associations ${ }^{(20,37)}$. Although the preliminary results of our study indicate that children's ethnicity does influence the associations between parental feeding styles and children's dietary intakes, further research is needed to refine our understanding of the detailed mechanisms involved here.

\section{Strengths and limitations of the study}

An important strength of the present study is that the sample of young children (with an average age of about 5 years) investigated was larger than in other studies of the association between parental feeding style and children's dietary intake. This made it possible not only to confirm the findings of previous studies with smaller samples and children of different ages, but also to examine the effect of maternal educational level and children's ethnicity on the results.

The use in the present study of data from the 'Kindermonitor' questionnaire, a routine management tool in Dutch youth health care, led to some limitations. First, 3-year-old children were over-represented in the 'Kindermonitor' sample as a consequence of creating sufficient power in this particular age group. However, other age groups were still large enough to be representative for the Dutch population as a whole. Post hoc analysis showed that associations between parental feeding styles and dietary intakes in 3 -year-old children were comparable with those in children aged 6-7 years. However, the association with 'instrumental feeding' was slightly stronger for 3-year-old children, while that with 'encouragement' was slightly stronger for 6- to 7-year-old children. The over-representation of 3-year-old children in our sample could thus possibly lead to overestimation of the association with 'instrumental feeding' in the group as a whole. Furthermore, our study sample was representative of the general Dutch population with regard to ethnicity, but mothers with high educational levels were over-represented (56.4\%). This may affect the generalizability of our results. Second, the standardized questionnaire used was developed as a generalized health monitoring tool and thus goes into relatively little detail on children's dietary behaviour. Hence, children's actual dietary intakes might deviate from the reported results. Third, the PFSQ questionnaire has not been validated for use in non-Dutch ethnic groups in the Netherlands. However, the fact that the PFSQ is widely used in other countries, including some outside Western Europe ${ }^{(38,39)}$, gives a strong indication of its general validity. A fourth limitation is that the crosssectional approach used in our study means that causal relationships between parental feeding styles and dietary intakes could not be determined. Finally, the reliance on self-reported data provided by parents means that responses might be biased to fit in with socially acceptable norms.

\section{Conclusion}

The present study extends our knowledge of the influence of parental feeding styles on young children's dietary intakes and provides a first indication of how this influence varies between specific subgroups. It shows that both controlling and encouraging feeding styles promote healthy dietary behaviour in children by raising the consumption of vegetables and reducing that of SSB. An encouraging feeding style is also related to higher intakes of fruit and water. In contrast, using food to modify a child's behaviour ('instrumental feeding') is shown to promote relatively unhealthy dietary behaviour in children by reducing the consumption of fruit, vegetables and water, and raising that of SSB.

The present study also explored the impact of maternal educational level and children's ethnicity on the associations between parental feeding styles and children's dietary intakes. 'Control' was found to improve children's vegetable consumption for all maternal educational levels and child ethnicities, but this effect was more pronounced in some cases. 'Encouragement' stimulates fruit and water consumption at all maternal educational levels and in non-native/non-Western and native Dutch children, and leads to a reduction in SSB consumption in children whose mother has a medium educational level and in native Dutch children. 'Instrumental feeding' tends to have an unfavourable effect on children's diet at most maternal educational levels and child ethnicities. 'Emotional feeding' shows few moderated associations; however, for these particular subgroups this feeding style is mainly beneficial.

There is a need for further investigation of the role of educational level and ethnicity in modifying the relationships between parental feeding styles and children's dietary intakes. It might also be interesting to explore the extent to which the prevalence (high level) of the various 
feeding styles is associated with meeting dietary recommendations. However, such investigations would require more information than is available at present on the cut-off points of parental feeding styles (high $v$. low).

The present study's findings could provide a basis for the development of interventions aimed at improving parental feeding practices. Such interventions might be useful across the board to hone parenting skills in connection with encouraging and controlling feeding styles, but in some cases it will be important to take maternal educational level and children's ethnicity into account. For example, interventions aimed at reducing SSB consumption may help mothers with high educational levels and native Dutch children, whereas school policies may be more effective in reducing SSB consumption in other subgroups.

\section{Acknowledgements}

Financial support: This research received no specific grant from any funding agency in the public, commercial or notfor-profit sectors. Conflict of interest: None of the authors had a conflict of interest. Authorship: S.Y.M.M. designed and conducted the study. M.-B.M.R.I. performed the statistical analyses, supervised by S.Y.M.M. C.M.R. had the general supervision. M.-B.M.R.I. led the writing of the manuscript, supervised by S.Y.M.M. and C.M.R. All authors had a substantial contribution to the manuscript and had final approval of the submitted version. Ethics of human subject participation: Data collection for the survey formed part of routine youth health care in the Netherlands and therefore did not require approval of the medical ethics review board.

\section{References}

1. Lock K, Pomerleau J, Causer L et al. (2005) The global burden of disease attributable to low consumption of fruit and vegetables: implications for the global strategy on diet. Bull World Health Organ 83, 100-108.

2. Schwingshackl L, Hoffmann G, Kalle-Uhlmann $\mathrm{T}$ et al. (2015) Fruit and vegetable consumption and changes in anthropometric variables in adult populations: a systematic review and meta-analysis of prospective cohort studies. PLoS One 10, e014084.

3. Mikkilä V, Räsänen L, Raitakari OT et al. (2004) Longitudinal changes in diet from childhood into adulthood with respect to risk of cardiovascular diseases: the Cardiovascular Risk in Young Finns Study. Eur J Clin Nutr 58, 1038-1045.

4. Kelder SH, Perry CL, Klepp KI et al. (1994) Longitudinal tracking of adolescent smoking, physical activity, and food choice behaviors. Am J Public Health 84, 1121-1126.

5. Van der Klauw M, Verheijden MW \& Slinger JD (2013) Monitor Convenant Gezond Gewicht 2013. TNO 2013 R12037. publications.tno.nl/publication/34617176/UkwPrt/ klauw-2013-monitor.pdf (accessed May 2015).

6. Voedingscentrum (2011) Richtlijn voedselkeuze. http:// www.voedingscentrum.nl/Assets/Uploads/voedingscentrum/ Documents/Professionals/Voedselvoorlichting/01_Richtlijnen voedselkeuze Voedingscentrum.pdf (accessed May 2015).

7. Malik VS, Schulze MB \& Hu FB (2006) Intake of sugarsweetened beverages and weight gain: a systematic review. Am J Clin Nutr 84, 274-288.
8. Davison KK \& Birch LL (2001) Childhood overweight: a contextual model and recommendations for future research. Obes Rev 2, 159-171.

9. Rodenburg G, Oenema A, Kremers SPJ et al. (2012) Parental and child fruit consumption in the context of general parenting, parental education and ethnic background. Appetite 58, 364-372.

10. Sleddens EF, Kroeze W, Kohl LF et al. (2015) Determinants of dietary behavior among youth: an umbrella review. Int $J$ Behav Nutr Phys Act 12, 7.

11. Ventura AK \& Birch LL (2008) Does parenting affect children's eating and weight status? Int J Behav Nutr Phys Act 5, 15.

12. Sleddens EFC, Gerards SMPL, Thijs C et al. (2011) General parenting, childhood overweight and obesity-inducing behaviors: a review. Int J Pediatr Obes 6, e12-e27.

13. Hoerr SL, Hughes SO, Fisher JO et al. (2009) Associations among parental feeding styles and children's food intake in families with limited incomes. Int J Behav Nutr Phys Act 6, 55.

14. Sleddens EFC, Kremers SPJ, De Vries NK et al. (2010) Relationship between parental feeding styles and eating behaviours of Dutch children aged 6-7. Appetite 54, 30-36.

15. Wardle J, Sanderson S, Guthrie CA et al. (2002) Parental feeding style and the inter-generational transmission of obesity risk. Obes Res 10, 453-462.

16. Van Der Horst K, Oenema A, Ferreira I et al. (2007) A systematic review of environmental correlates of obesity-related dietary behaviors in youth. Health Educ Res 22, 203-226.

17. Pearson N, Biddle SJH \& Gorely T (2009) Family correlates of fruit and vegetable consumption in children and adolescents: a systematic review. Public Health Nutr 12, 267-283.

18. Lo K, Cheung C, Lee A, Tam WWS et al. (2015) Associations between parental feeding styles and childhood eating habits: a survey of Hong Kong pre-school children. PLOS One 10, e0124753.

19. Saxton J, Carnell S, van Jaarsveld CHM et al. (2009) Maternal education is associated with feeding style. J Am Diet Assoc 109, 894-898.

20. Raaijmakers LG, Gevers DW, Teuscher D et al. (2014) Emotional and instrumental feeding practices of Dutch mothers regarding foods eaten between main meals. BMC Public Health 14, 171.

21. Korzilius H (2008) De kern van survey - onderzoek. Assen: van Gorcum.

22. Bessems KM, van Assema P, Martens MK et al. (2012) Healthier food choices as a result of the revised healthy diet programme Krachtvoer for students of prevocational schools. Int J Behav Nutr Phys Act 9, 60.

23. Rodenburg G, Kremers SPJ, Oenema A et al. (2014) Associations of parental feeding styles with child snacking behaviour and weight in the context of general parenting. Public Health Nutr 17, 960-969.

24. Gliem JA \& Gliem RR (2003) Calculating, interpreting, and reporting Cronbach's alpha reliability coefficient for Likerttype scales. Presented at Midwest Research-to-Practice Conference in Adult, Continuing, and Community Education, Ohio State University, Columbus, OH, 8-10 October 2003; available at http://www.ssnpstudents.com/ wp/wp-content/uploads/2015/02/Gliem-Gliem.pdf

25. Statistics Netherlands (2017) Standaard onderwijsindeling 2016. https://www.cbs.nl/-/media/_pdf/2017/13/pubsoi2016ed1617. pdf (accessed January 2017).

26. Statistics Netherlands (2000) Standaarddefinitie allochtonen. Hoe doet het CBS dat nou? pp. 24-25. https://www.cbs.nl/ $\mathrm{nr} /$ rdonlyres/26785779-aafe-4b39-ad07-59f34dcd44c8/0/ index1119.pdf (accessed January 2017).

27. Field A (2005) Discovering Statistics Using SPSS, 2nd ed. London: SAGE Publications Ltd.

28. Ocke MC, van Rossum CTM \& Fransen HP (2007) Dutch National Food Consumption Survey - Young Children 
2005/2006. http://www.rivm.nl/bibliotheek/rapporten/35007 0001.html (accessed June 2015).

29. Sleddens EFC, Kremers SPJ, Stafleu A et al. (2014) Food parenting practices and child dietary behavior. Prospective relations and the moderating role of general parenting. Appetite 79, 42-50.

30. Nicklaus S (2009) Development of food variety in children. Appetite 52, 253-255.

31. Jones LR, Steer CD, Rogers IS et al. (2010) Influences on child fruit and vegetable intake: sociodemographic, parental and child factors in a longitudinal cohort study. Public Health Nutr 13, 1122-1130.

32. Maccoby EE \& Martin JA (1983) Socialization in the context of the family: parent-child interaction. In Handbook of Child Psychology. vol 4: Socialization, Personality, and Social Development, pp. 1-102. New York: John Wiley \& Sons, Inc.

33. Benton D (2004) Role of parents in the determination of the food preferences of children and the development of obesity. Int J Obes Relat Metab Disord 28, 858-869.
34. Kröller K \& Warschburger P (2009) Maternal feeding strategies and child's food intake: considering weight and demographic influences using structural equation modeling. Int J Behav Nutr Phys Act 6, 78.

35. Birch LL (1999) Development of food preferences. Annu Rev Nutr 19, 41-62.

36. Kröller K, Jahnke D \& Warschburger P (2013) Are maternal weight, eating and feeding practices associated with emotional eating in childhood? Appetite 65, 25-30.

37. Larsen JK, Hermans RCJ, Sleddens EFC et al. (2015) How parental dietary behavior and food parenting practices affect children's dietary behavior: interacting sources of influence? Appetite 89, 246-257.

38. Tam W, Keung V, Lee A et al. (2014) Chinese translation and validation of a parental feeding style questionnaire for parents of Hong Kong preschoolers. BMC Public Health 14, 1194.

39. Ozcetin M, Yilmaz R, Erkork U et al. (2010) Reliability and validity study of parental feeding style questionnaire. Turk Pediatr Ars 45, 124-131. 